The Pictor Technique:

Exploring experiences of collaborative working from the perspectives of generalist and specialist nurses

Nigel King, Joanna Brooks, Alison Bravington, Beth Hardy, Jane Melvin and David Wilde. 


\section{INTRODUCTION: THE PICTOR TECHNIQUE}

Interviews are widely used in qualitative research, and can take many different forms (see King and Horrocks, 2010). In this chapter, we will introduce a particular visual tool developed by the authors and colleagues for use in research interviews: the Pictor technique (e.g. King, Bravington, Brooks et al. 2013). The technique requires research participants to construct a 'Pictor chart' - a visual representation depicting their role and their work - which the researcher and participant can then use as a basis to explore potentially complex experiences in a research interview setting. We will present here an example from our own applied research to demonstrate how we have used Pictor in research interviews.

\section{Doing Pictor}

Before introducing our case example, let us first familiarise ourselves with the main procedural steps undertaken in the technique. Pictor was initially developed from a technique used in family therapy (Hargreaves, 1979) and first used in research with health and social care professionals (Ross, 2005; Ross et al., 2005) ,It has also been successfully adapted for use with patients and carers, looking at their experiences of being in receipt of multiple care services (Hardy, King and Firth, 2012). Research using Pictor has a primary focus on 'collaborative working', which we have previously defined as occurring when two or more people from different professional groups are required to interact to achieve a shared objective (King et al., 2013). In health and social care settings, this shared objective is usually the delivery of appropriate care to a service user.

Below, we provide some generic guidelines for implementing the technique. However, it is important to remember that these are intended to be flexible, and that the primary aim of Pictor is to facilitate reflection and discussion with the research participant. Modifications may be necessary and appropriate when using the approach with different types of participant as King, Bravington, Brooks et al. (2013) illustrate. 
1. First, the researcher asks the research participant to recall a case in which they and others were required to work together to achieve some goal or purpose that fits the criteria of the particular research question.

2. The participant is next asked to think of all the people involved in this case in any way. This must include the participant him or herself but there are otherwise no restrictions placed on who the participant may choose to include. Participants should be encouraged to think as widely as possible. The participant is then provided with a stack of arrow shaped sticky notes and asked to label these with a pseudonym and/ or a role title for each person on a separate note. The notes we use come in three different colours and participants are informed that they are free to use these colours to make distinctions if they so wish, but that there is absolutely no necessity to do so.

We have sometimes found that participants want to use notes to refer to groups rather than individuals (for example, 'social services domestic team' rather than 'Sandra'). Unless the study specifically required only individuals to be represented, there would be no problem with this. Similarly, notes may be used to refer to other factors identified as playing an important role in a case aside from human agents (family pets have made several appearances in our work, as has the weather!).

3. Once the participant has generated their labelled arrows, they are asked to place these on a large sheet of paper in a way which helps them represent the case. This representation is the participant's Pictor chart. Participants are able to use proximity or direction of the arrows to represent aspects of roles and relationships in the particular case if they so wish, but there are no fixed rules about how this should be done; they are encouraged to design their chart in whatever way they can best represent the case in mind. There are no time constraints on chart construction, but participants rarely need longer than around fifteen minutes to produce even quite a complex chart. 
4. Once the Pictor chart is completed, the participant is asked to talk the interviewer through the story of the case, with the chart serving as the focus for discussion. The interviewer can then use prompts based on the layout of the arrows - for example, it might be appropriate to ask why some arrows are closer together than others, whether the direction of the arrows represents anything particular about the case, or whether different arrow colours have been used in any systematic way.

If, in the course of the discussion, the participant realises that there are additional arrows they would like to add to their chart, they are encouraged to do so. Sometimes, in the course of reflecting on the case, participants want to alter the layout or move the arrows to represent changes over time. This is not problematic, but the interviewer should record where the arrow was originally placed by marking this original position with a dotted line.

5. There are no set rules regarding whether the interviewer should be present whilst the Pictor chart is being created. Our original practice was to give research participants clear instructions with regards to constructing their chart and to audio record this stage. We then left participants alone so as to give them clear autonomy over the creation of their chart, and to prevent them feeling overlooked. Once the participant had completed their chart, the interviewer would then reenter and recommence audio-recording for the discussion. However, some participants may be more unsure about constructing their chart and this may be dependent on the particular participant group being interviewed - for example, in our research, lay people - patients and carers - could be less confident than professionals in this respect). The interviewer can remain with participants if this is appropriate (in this case, audio recording should continue, as participants may wish to discuss their emerging chart as they construct it) but musttry to avoid 'leading' the participant in any way, even implicitly.

6. The final stage of data collection, immediately after each interview, is to create a record of the Pictor chart. The interviewer should draw around each arrow, copying the label written by the participant. The colour of the arrow should also be recorded. The interview date and codes to identify the participant and the 
interviewer are noted on the chart. Finally, the interviewer should take a goodquality digital image of the chart.

7. Analysis of data from Pictor interviews will depend on the overall methodological approach of the study within which the technique was used. Thus, for example, if Pictor was used in a study that took a narrative approach a suitable form of narrative analysis would be chosen to analyse the participant's discussion of their chart with the researcher, whilst a phenomenological study would look at the account in terms of how it illuminated participants' lived experience of the phenomenon under investigation.. Whatever the methodological approach taken, we would encourage researchers to include the Pictor charts as part of the data set for a study. Looking at the chart can help give a sense of where the participant sees him- or herself in relation to the others included in the chart. Close scrutiny of the chart may suggest fruitful directions for textual analysis, as we will illustrate in our example below.

Especially in studies where a considerable number of charts are collected, it can be useful to look at patterns of commonality and difference across charts as part of the analytic process. If some groups of participants consistently include or exclude certain people/agencies, place them in particular areas of the chart, or lay out their arrows in particular configurations, this can invite the researcher to examine the data more closely to see what the patterns may mean. In Hardy's 2012 study of patients' and their partners' experiences of receiving services for advanced disease, for example, she found that General Practitioners (GPs) were very often placed in a peripheral position on charts, or not included at all. Looking at what participants said about GPs then revealed some important perceptions about their role in care (Hardy, 2012).

As well as being a powerful tool for eliciting and exploring peoples' accounts of collaborative working, the Pictor technique has a real strength for the dissemination of research findings. We have found in numerous conference papers, workshops and seminars that examples of real charts from our studies provides an effective way of engaging both academic and practitioner audiences with key messages from our work. 


\section{OUR CASE EXAMPLE}

\section{Introduction to the study}

We will now use an example from our own research (King et al., 2013) to illustrate how Pictor can be usefully applied in real world research settings. Our example is taken from a project undertaken in the United Kingdom over three years, funded by Macmillan Cancer Support. The primary focus of the research was to examine how different types of nursing professionals worked with each other, with other professionals and with patients and carers to support patients with cancer. Effective collaborative working between different professionals is a crucial part of contemporary health and social care, and essential to the provision of good quality care for patients and their families. In this project, we focused on how different professionals involved in the care of patients understand their own role and the roles of others, and how these understandings impact on inter-professional relationships across different care settings on the provision of patient care. As well as examining what factors might help or hinder collaborative working for nurses, we were also interested in comparing experiences of collaborative working related to cancer patients with experiences relating to patients with long term conditions such as heart failure and diabetes. We hoped the findings of our study would highlight areasfor the further development of services to support patients with a past or current diagnosis of cancer.

The research was set in one mainly urban metropolitan borough in the north of England with high levels of deprivation and health inequalities. This was, in qualitative terms, a large scale project, and we interviewed a total of seventy-nine participants. Interviews were conducted using Pictor to explore experiences of collaborative working from the different perspectives of our various participants.

\section{Why was Pictor the appropriate method to use?}

There are particular methodological challenges associated with exploring experiences of collaborative working with healthcare professionals. Often, cases are very complex involving many different individuals, and it can be difficult for research 
participants to recall all those involved when questioned about this in a research setting. This can lead to a tendency to include only 'key' players, omitting those with a more peripheral but nonetheless important role to play. Healthcare professionals are often very aware of policy rhetoric around the importance of good collaboration, and when asked about their own involvement in and understanding of collaborative working, they can sometimes present an 'official' explanation or ideal version rather than a direct account of their own experiences. Additionally, for experienced professionals, the way they work with others may have become so habitual and ingrained that it can be difficult for them to reflect on in any depth when questioned by an interviewer. Pictor provided us with an effective technique to help overcome these challenges in our research.

Pictor has its intellectual origins in Personal Construct Theory (PCT; Kelly, 1955), and particularly in phenomenological readings of PCT by more recent scholars (e.g. Butt, 2003). Our thinking about professional roles, relationships and identities in the design of this study was informed by constructivist understandings of the person, and especially by both PCT and by interactionist views on the nature of professions and professional identities (e.g. Macdonald, 1995). In both these traditions, a person's sense of who they are and what they do as a professional are seen as being shaped through their interaction with the social world they inhabit. An understanding of 'self as professional' is something that is achieved through practice, and especially through relationships with fellow professionals, patients and their families. According to PCT, human beings are seen as meaning makers, formulating personal hypotheses or constructs to explain their worlds. This process of meaning making, referred to in PCT as 'construing' is not a rationalistic mental operation(Warren, 1990), and happens not 'inside' the person but through his or her actions and interactions in and with the world (e.g. Butt, 1998; Walker and Winter, 2007). From a PCT perspective, we are often not consciously aware of our construing. Much of it becomes taken for granted and accepted as simply the "natural" way of seeing ings, rather than as a perspective that can be altered. In this piece of research, in which we sought to facilitate reflection on collaborative working practices, this approach and our utilitsation of Pictor were thus an excellent fit. 


\section{APPLYING THE PICTOR TECHNIQUE}

Here we will describe how we applied Pictor in the study described above. We will cover data collection using Pictor, how we drew on the visual material in our analysis, and conclude by highlighting some of the lessons we learned about the technique from its use in this study.

\section{Data collection with Pictor}

Our core participant group in this project was made up of generalist and specialist nurses who worked with cancer and/ or long-term condition patients, but we also recruited a substantial number of participants from other professional groups (including managers, General Practitioners and social services staff), as well as patients and lay carers. All interviews undertaken with professionals were held in private and quiet settings within participants' workplaces. Interviews with patients and carers were held in their own homes.

We started the interviews with professionals by asking them to describe their own role, and to provide some background information about their professional experience and career history. Then, participants were asked to bring to mind a case of collaborative working they remembered clearly to create their Pictor chart. Where possible, if their role included working with different patient groups, professional participants were asked to construct two Pictor charts: one depicting a case involving a cancer patient and another involving a long-term condition patient. If this were not possible, they were asked for one case in their area of practice. Those who did not have clinical roles (e.g. managers) were asked to provide examples of specific collaborative projects. In interviews with patients and lay carers, the focus was on the patient's own case. In all cases, participants were asked to discuss the roles and relationships amongst those involved, using the Pictor charts to focus their reflection and the interviewer's questioning. 


\section{Analysis of Pictor-based data}

Interviews were audio-recorded and transcribed verbatim. We could have simply analysed the textual data in the transcripts with no further reference to the Pictor charts. Such a strategy would in effect treat Pictor as just a method of eliciting rich textual data - a worthwhile endeavour in itself. However, we take the view that the Pictor charts should be seen as data in their own right, and that analysis can benefit from a consideration of both the textual and visual data in relation to each other. In the present study, we used template analysis (King, 2012) for the textual data; we will not describe this in detail here (see King et al, 2013, for more information) but rather will concentrate on how we drew on the visual data in the Pictor charts. We did this in two main ways, which might be termed "within-participant" and "acrossparticipant" strategies.

"Within-participant" analysis involves moving between examination of the chart and the text to build up an understanding of the participant's experience. In practical terms this meant having the chart(s) produced by a participant in front of us as we went through the steps of coding in template analysis. We would note distinctive features of a chart and turn to the transcript text to see how it shed light on these; for example, we might be surprised to see a particular professional placed in a very peripheral position on the chart, or even to find them absent. This would prompt us to pay attention in the transcript to what the participant said about working relationships with this individual and/or professional group.

"Across-participant" analysis involves consideration of multiple charts, looking for similarities and differences between them that might tell us something of interest about the data as a whole. The kinds of features that drew our attention in this study included the overall shape of charts, the total number of arrows used, and whether and where particular groups tended to be represented. This is not an exhaustive list, nor is it intended to be prescriptive -the focus of across-participant analysis needs to reflect the aims and objectives of a particular study. Bravington (2011), for example, provides a useful account of how charts were used across participants in a study 
examining how nursing and midwifery students reflected on collaborative working in their placements.

\section{Illustrative findings}

Given that the focus of this chapter is on Pictor as a method rather than the substantive topic of collaborative working, we will not seek to provide a comprehensive overview of the study findings here. Instead, we will present one Pictor case, displaying the chart and summarising the content of the discussion about it between interviewer and interviewee. We will then use the chosen case to illustrate the kind of things we do in our "within-participant" analysis, before presenting some aspects of the "across-participant" analyses in this study.

INSERT FIG 1: PICTOR EXAMPLE - NETWORK CHART

This case is taken from an interview with 'Martha' - a Staff Nurse on a Cardiology ward at the District General Hospital at the centre of the borough. It relates to a 67 year-old woman ('Angela') who had a long history of heart failure, with a number of other health problems. Angela had been admitted to the ward many times.

On the occasion in question, Angela was admitted feeling "quite unwell" and required input from a range of services, including the pain team, specialist heart failure nurses, gastrology and haematology consultants, and district nurses. The major intervention, though, was the replacement of a heart valve, which required referral to a cardio-thoracic surgeon at a larger hospital outside the district. The ward staff had got to know Angela and her family well over the years; Martha describes the family as very supportive, but also notes that they themselves required a good deal of support from the nursing staff; 
"...obviously the family was quite a big input as well because they were quite a close knit family, there was her and her husband who lived and managed together, outside family like daughters and grandchildren and that, but he was one of these little doting men, wanting to know everything, really anxious, he needed a lot of support, they needed a lot of support as well from that side of it."

Angela had her surgery at the outside hospital, and returned to Martha's ward for further care and recuperation. The entire episode from admission to her discharge home took about six weeks, which - given the complicated nature of her condition Martha felt to be "very quick". Overall, she described collaborative working between professionals (including across organisations) and with patient and family as highly effective;

"I think everything went smoothly for her coming back, everything was dealt with really, really well, from all types of levels. Family were involved and kept up to date with everything so they knew what was going on, there wasn't an instance where they ever felt, oh they didn't know where they were up to"

On her chart Martha placed the arrow for "Patient" in the middle and towards the top of the chart. Her own arrow ("Nurse") and that for "Family" are touching the patient arrow, with those for "District Nurses" and "Specialist Nurse" close on one side. A little further out are "Pain Team" on one side and "Physio + O.T." (physiotherapist and occupational therapist) together on one arrow fairly close on the other side. The four different consultants are placed further down in a line, with "Pharmacy" in the middle behind them. 
When starting to analyse an individual Pictor chart and the accompanying interview, the first step is for researchers simply to familiarise themselves with the 'story' by reading the relevant section of the interview carefully, and looking at the overall layout of the chart.Are the arrows laid out in any particular pattern - such as a circle, a series of ranks, a single long line (or combination of such shapes) - and does this seem to coincide with points made in the discussion? Looking more closely, the following features may be worth attending to (although this is not by any means an exhaustive list):

- Proximity - how close are arrows to each other? Which are especially close or placed a long way from the rest?

- Colour - if different colour arrows were available, does the participant appear to have used them in a systematic way?

- Direction- might arrow direction indicate something of significance in the participant's story? For instance, is there one that stands out as being in the opposite direction to others around it?

- Grouping - Do there appear to be different groupings of arrows in the chart?

In Martha's example, the chart appears to have two main groupings that differ in shape and proximity to the patient - a circle of arrows around the patient, and a straight line underneath this circle. As noted above, the "agents" represented in these two parts are of different types - mostly nursing groups in the close circle, and consultants in the line. The separation in shape is reinforced by Martha's use of colour. The sticky arrows we used came in three different colours, which we have noted on the figure. Martha has used yellow arrows to represent patient and family, orange for all the nurse groups (and the physiotherapist/occupational therapist), and green for the consultants and pharmacy. The interviewer checked with Martha whether she had a particular purpose in mind in her use of arrow colour; 
“Interviewer: Right then l've noticed you've used three colours have you used them to represent anything in particular

Martha: Just like the nursing side and like there's physio, so they're like the main team, the darker green is more like the consultants more of the medical side and the patient's side is the yellow colour."

The division into three "sides" is thus quite explicit, and it is worth noting that Martha describes the nurses as "the main team". The way Martha has used grouping, proximity to the patient, and colour thus helps convey the way she sees Angela's story of care - that her main support is through the nurses who look after her on the ward (and beyond), while the various medical interventions are temporary intrusions into her experience of care. This is not to suggest that Martha does not value what the consultants do; indeed, she is consistently positive about them. Nevertheless, the image is one that reverses the stereotypical view of nurses supporting doctors; here the doctors are literally depicted as a "back line" supporting the main team of nurses.

One further point to note in Martha's case is how she represents herself. On the chart, she does this in the form of the arrow immediately underneath that for the patient, labelled "Nurse". However, in the discussion of the chart, she almost always refers to herself as part of the ward nursing team, rather than as an individual:

"[Angela] had surgery and came back to us post-operatively, was quite, really unwell when she came back, we thought maybe there was a possibility she wouldn't improve..."

"...so we were like using infusions to get rid of her oedema and we were also keeping her quite anticoagulated because we weren't using the Warfarin. Pain 
control was good, specialist nurse input was only like heart failure at that time, we didn't need anything else."

We have found across several studies that nurses who work in a strongly teamoriented way often present themselves collectively rather than individually, which frequently includes the use of a team rather than individual arrow on the chart. Martha's example highlights the importance of looking closely at the transcribed discussion when considering the chart, and not jumping to conclusions just from the visual material; her use of the singular "Nurse" on the arrow does not indicate any lack of a team perspective in how she saw her role in the case.

\section{Across-participant analysis}

Looking at the Pictor charts from different groups of nurses in this study, one very striking difference was apparent. In the data set as a whole, the most common configuration is what we refer to as a "network" chart, where the main organising principle used by the participant is to use the arrows to depict the nature and/or quality of relationships between themselves, the patient and family, and other professionals. (Often these also highlight aspects of relationships amongst other agents on the chart too.) Across a range of studies we have conducted using Pictor, the network form is by far the most common, and in this study this was the case for most of the nursing groups. However, for acute-based (i.e. hospital) specialist nurses the most common configuration was the "timeline". In this, arrows are laid out to represent a temporal sequence of events and actions, perhaps with branches to the side to show where several other professionals and/or lay people had an involvement in the case. (An example of a timeline format chart is shown in figure 2).

INSERT FIG 2: PICTOR EXAMPLE - TIMELINE CHART 
Typically, the patient, the professional constructing the chart, and other key players are depicted several times along the timeline. Looked at in percentage terms, $72 \%$ of community nurse charts were networks and $21 \%$ timelines (the remainder used a mixture of the two types). In contrast, $33 \%$ of acute specialist nurses' charts were networks and $61 \%$ timelines.

Why might acute specialist nurses be much more likely than community nurses to use timeline format charts? To answer this, we needed to go back to look at the individual interviews and charts, especially those of the acute specialist nurses, in detail. One potential influence could be their experience of reflective methods as part of their specialist training. We had not systematically asked participants about this, nor did we have extensive data with regards to post-registration training. However, indirect evidence tends to make us sceptical that past training in reflection was likely to have been a major factor. None of the participants made any reference to other reflective techniques with which they were familiar, neither to highlight similarities to nor differences from - Pictor. It is unlikely, given their evident variation in age and tenure, that all the acute specialist nurses who used timeline charts went through the same training regime at the same time. Furthermore, if specialist nurse training in general facilitated the timeline style, we would also expect to see it prominently amongst the charts produced by community specialist nurses, but we do not.

A further possible explanation for the prominence of timeline-format charts could lie in the nature of the acute nurse specialist's role. Their involvement with patients is generally episodic; they see patients for check-ups or assessments related to interventions, and when there is a crisis relating to their condition. This may encourage the nurses to conceptualise cases as a series of distinct steps or phases in the trajectory of an illness. Regarding their engagement with other professionals, the charts and interviews show that this is often with other specialist nurses, and Consultants and their teams. Commonly, these contacts are for the purpose of referring the patient on for a particular intervention, or seeking advice and information in relation to the urgent situation for which they have consulted. This contrasts with community-based nurses, who tend to describe a wider range of types 
of interaction with a wider range of other professionals/services. Such a pattern may tend to favour timeline formats for acute specialist nurses but networks for community-based nurses.

The kind of analysis we have described here may appear to be equivalent to the quantitative approach to examining social networks commonly used in some forms of social network analysis (e.g. Patterson, Pfeiffer, Weaver et al, 2013). It differs, however, in that when we analyse patterns across participants in Pictor we do not seek to determine objective features of networks that may be summarised statistically. Rather, we use the emerging patterns to raise questions as to what may be going on in participants' experiences. To answer these questions we need to return to the textual data analysis and to consideration of individual Pictor charts in the context of their interview as a whole.

\section{Reflections on using Pictor}

This is our largest study to date incorporating Pictor, and it has offered us an important opportunity to learn how best to use the technique. It has confirmed that the great majority of participants are able to follow the technique without trouble, and many commented that they found it interesting and enjoyable to do.. The only groups from which participants refused to use the technique were patients and carers, which accords with our previous experience that lay people may find it a little more challenging and/or unfamiliar than professionals - but even here the clear majority were happy to do it.

The fact that we collected a large number of charts gave us our best chance yet to consider the value of cross-participant analyses based primarily on the charts. This proved very worthwhile, enabling some important insights into differences between nursing groups' experiences of collaborative working. We were also able to compare experiences of collaborative working by disease type, since we collected two charts from many participants - one based on a cancer case and one on an LTC. We do not have the space to discuss this in detail here, but the overall picture was that 
professionals' experiences of the process of collaboration did not appear to vary greatly according to the type of condition, but there were differences in the extent to which there was collaboration across the acute/community sectors.

The project, which collected a large volume of Pictor chart and interview data, highlights the importance of being systematic and well-organised in managing data. It would be all too easy to waste a great deal of time matching anonymised charts to audio files, identifying which participant group a chart came from, and so on. We devised a coding system enabling us to identify each chart and audio file by participant type, illness condition and the identity of the interviewer. . Even in a much smaller study we would strongly recommend that this kind of system is designed in advance of data collection.

Looking critically at the way we used Pictor in this study, one change we would make in further studies would be to ask participants more questions about the chart itself than we did in some of these interviews. We tended to do so mainly where some aspect of chart construction seemed important to the story the participant was telling, and sometimes did not ask about details that appeared more peripheral. For example, in Martha's case, we did not ask about how the O.T. and physiotherapist were involved, and why they were included in the "main team" (as represented both by proximity and arrow colour) who otherwise were all nurses. Of course, there is a balance to be struck here; to go through every aspect of a chart with each participant could interfere with the goal of enabling them to tell the story of their case in their own way. Nevertheless, we feel that it would be fruitful to focus a little more consistently and systematically on the chart itself in future.

In conclusion, the Pictor technique has much to offer to qualitative psychologists whose research addresses issues related to collaboration between different professional groups. While our work has concentrated on health and social care settings, there is nothing intrinsic to the technique which means it can only be applied there. Any area of activity where people with different outlooks, backgrounds and/or identities have to work together could be the focus of research using the Pictor technique; for example education, the criminal justice system and many private sector businesses. 


\section{References}

Bravington, A. (2011) Using the Pictor technique to reflect on collaborative working in undergraduate nursing and midwifery placements. Master's thesis, University of Huddersfield, United Kingdom. Available at http://eprints.hud.ac.uk/id/eprint/13720

Butt, T. W. (1998). Sociality, role and embodiment .Journal of Constructivist Psychology, 11(2), 105-116.

Butt, T. W. (2003). The phenomenological context of PCP. In F. Fransella (Ed.), International handbook of personal construct psychology (pp. 379-386). Chichester, UK: Wiley.

Hardy, B. (2012). "Everyone was like flies around a jam pot": a phenomenological study exploring the experiences of people affected by advanced disease in relation to the involvement of multiple health care services. Unpublished Ph.D. thesis, University of Huddersfield, United Kingdom. Available at: http://eprints.hud.ac.uk/17133/

Hardy, B., King, N., \& Firth, J. (2012). Applying the Pictor technique to research interviews with people affected by advanced disease. Nurse Researcher. 20(1), 610

Hargreaves, C. P. (1979).Social networks and interpersonal constructs. In P.

Stringer and D. Bannister (Eds.), Constructs of sociality and individuality (pp. 153175). London: Academic Press.

Kelly, G. A. (1955). The Psychology of Personal Constructs. New York: Norton. King, N. (2012) Doing Template Analysis. In G.Symon and C.Cassell (eds.) The Practice of Qualitative Organizational Research: Core Methods and Current Challenges. London: Sage.

King, N., Bravington, A., Brooks, J., Hardy, B., Melvin, J. and Wilde, D. (2013). The Pictor Technique: a method for exploring the experience of collaborative working. Qualitative Health Research, 23 (8), 1138-1152.

King, N. and Horrocks, C. (2010) Interviews in Qualitative Research. London: Sage. King, N., Melvin, J.; Brooks, J., Wilde, D. and Bravington, A., (2013). Unpicking the Threads: How specialist and generalist nurses work with patients, carers, other professionals and each other to support cancer patients in the community. End of Project Report, Macmillan Cancer Support. Available at: http://eprints.hud.ac.uk/18481/

Macdonald, K.M. (1995). The Sociology of the Professions. London: Sage. 
Patterson, D., Pfeiffer, A.J., Weaver, M.D., Krackhardt, D., Arnold, R.M., Yealy, D.M. and Lave, J.R. (2013). Network analysis of team communication in a busy emergency department. BMC Health Services Research, 13:109. http://www.biomedcentral.com/1472-6963/13/109

Ross, A. (2005). Collaborative working between district nurses and social workers. Unpublished $\mathrm{PhD}$ thesis, University of Huddersfield, United Kingdom.

Ross, A., King, N., \& Firth, J. (2005). Interprofessional relationships and collaborative working: Encouraging reflective practice. Online Journal of Issues in Nursing, 10(1), Art. 3. doi:10.3912/ojin.vol10no01man03

Walker, B. M., \&Winter, D. A. (2007). The elaboration of personal construct psychology. Annual Review of Psychology, 58, 453477.doi:10.1146/annurev.psych.58.110405.085535

Warren, W. (1990). Is personal construct psychology a cognitive psychology? International Journal of Personal Construct Psychology, 3(4), 393414.doi:10.1080/10720539008412828 
Figure 1. Example of a Pictor chart from "Martha" - staff nurse on cardiology ward

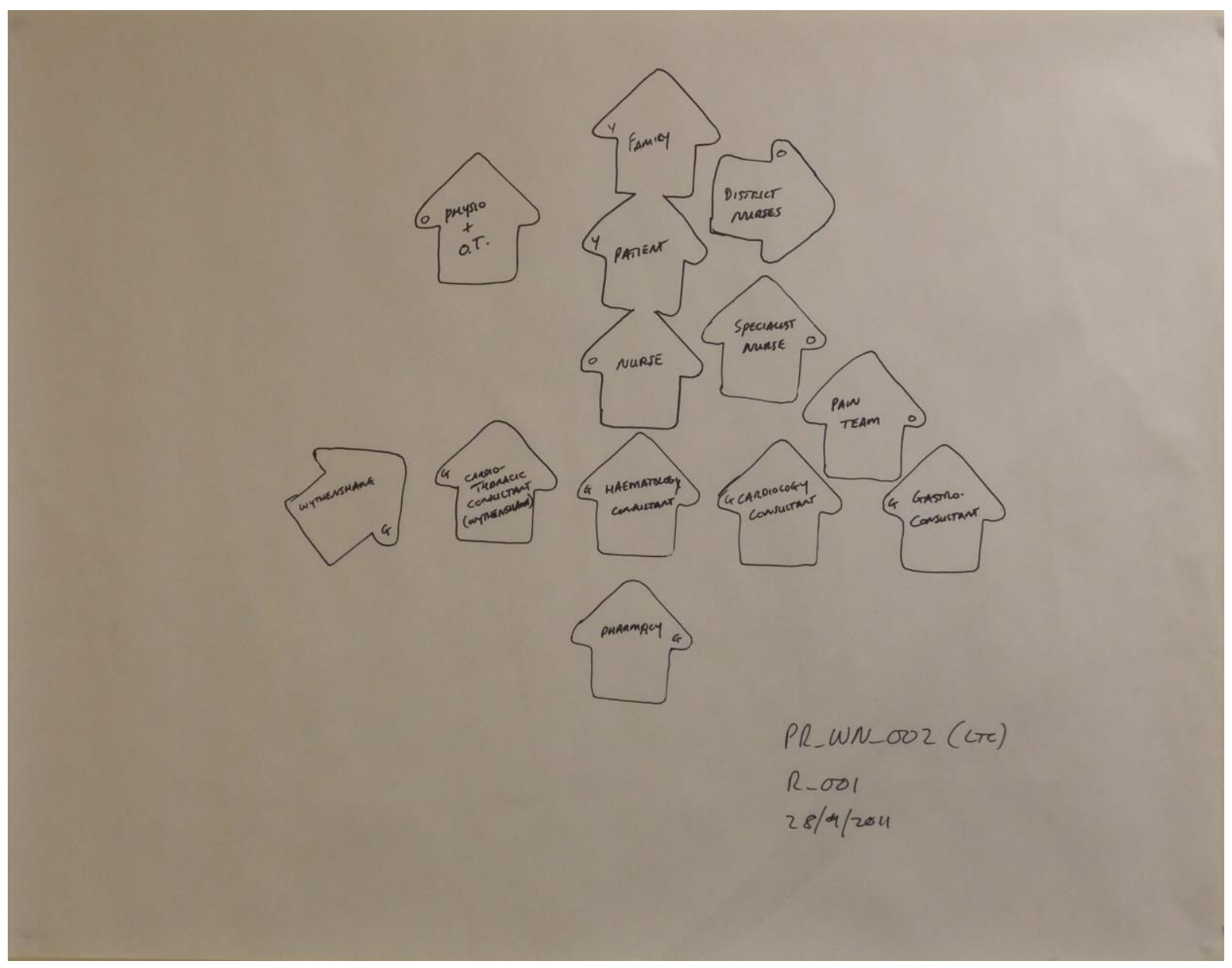


Figure 2. Example of a 'timeline' format Pictor chart

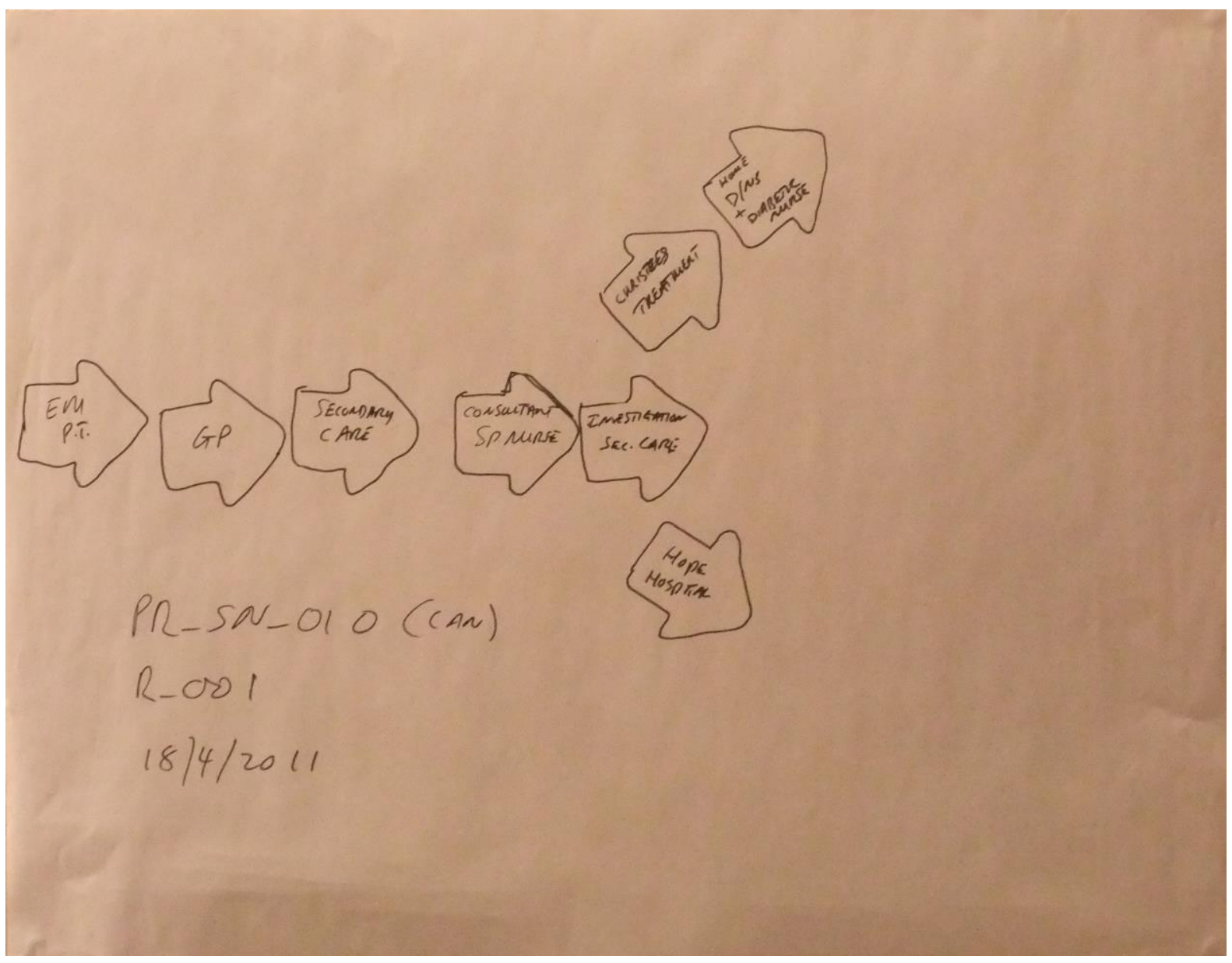

